\title{
THE MIRROR SYSTEMS OF INTEGRABLE EQUATIONS
}

\author{
JISHAN HU * AND MIN YAN $\dagger$
}

We provide an algorithm to convert integrable equations to regular systems near non-characteristic, movable singularity manifolds of solutions. We show how the algorithm is equivalent to the Painlevé test. We also use the algorithm to prove the convergence of the Laurent series obtained from the Painlevé test.

1. Introduction. A differential equation is said to have the Painlevé property if its solutions are single-valued near movable singularities [18] [20]. The property is very important because its close connection to the integrability and chaos [7] [16].

The study of the Painlevé property has a long history. In 1856, Birot and Bouquet [3] determined first order equations of the form $F\left(u^{\prime}, u\right)=0$ satisfying the Painlevé property. Later on, L. Fuchs [4], Poincaré, and Painlevé [17] extended the result to equations of the form $F\left(u^{\prime}, u, x\right)=0$. In 1887, Picard raised the natural question of the classification of equations of the form $F\left(u^{\prime \prime}, u^{\prime}, u, x\right)=0$ with the Painlevé property. The problem was settled by Painlevé [18] [19], Gambier [6], and R. Fuchs $[5]$.

The most widely used method for studying the Painlevé property has been the Painlevé test. The method has been used since more than a century ago [8] and played a key role in Sonia Kowalevskaya's monumental work [15]. It was formalized in [2] [20], and has been widely used as the test for the Painlevé property (and consequently, as the test for integrability). The test assumes a stronger version of the Painlevé property by requiring that the solutions must be meromorphic (thereby excluding single-valued essential singularities such as $e^{\frac{1}{x-x_{0}}}$ ) near movable singularities and then test the formal algebraic compatibility as a consequence of the requirement. Consequently, the relation between the Painlevé test and the Painlevé property is heuristic at best. For equations passing the Painlevé test, further rigorous proofs are needed in order to establish the Painlevé property [11] [18]. In fact, further proofs are necessary even for the convergence of the series obtained from the Painlevé test. Naturally, such proofs must contain some analytic ingredients that are missing in the Painlevé test.

The techniques such as inverse scattering transform may be used to find certain classes of solutions of integrable equations and can be then used to show that these

*Address: Department of Mathematics, The Hong Kong University of Science and Technology, Clear Water Bay, Kowloon, Hong Kong. Email: majhu@uxmail.ust.hk. Fax number: (852)23581643 .

$\dagger$ Address: Department of Mathematics, The Hong Kong University of Science and Technology, Clear Water Bay, Kowloon, Hong Kong. Email: mamyan@uxmail.ust.hk. Fax number: (852)23581643. 
solutions have Painlevé property [2] [16]. Besides this, there have been only two attempts (of more than 90 years apart !) at directly proving the Painlevé property: Painlevé's original proof [18] for the equation (the first of six Painlevé equations)

$$
u^{\prime \prime}=6 u^{2}+x,
$$

and Joshi and Kruskal's proposal [11] for the six Painlevé equations. As pointed out before, both approaches must rely on some analytic ingredients. In Painlevé's case, he converted the equation (1.1) into

$$
\left\{\begin{aligned}
\frac{d \theta}{d x} & =1+\frac{1}{4} x \theta^{4}+\frac{1}{4} \theta^{5}-\frac{1}{2} \xi \theta^{6} \\
\frac{d \xi}{d x} & =\frac{1}{8} x^{2} \theta+\frac{3}{8} x \theta^{2}+\left(\frac{1}{4}-x \xi\right) \theta^{3}-\frac{5}{4} \xi \theta^{4}-\frac{3}{2} \xi^{2} \theta^{5}
\end{aligned}\right.
$$

near movable singularities, where $u=\theta^{-2}$, and $\xi$ is essentially the resonance in the Painlevé test for (1.1). On the other hand, Joshi and Kruskal converted the six Painlevé equations into integral equations.

If we want to systematically investigate analytic properties for equations passing the Painlevé test, a natural thing we should try is to generalize the analytic ingredients in the two direct approaches above. Indeed, we will show in this paper that there is a general algorithm for finding a regular system like (1.2). We call such a regular system a mirror system of the original equation. In a subsequent paper [9], we will show how to find mirror systems for integrable systems.

We give two immediate applications of the mirror systems. The first is to use the regularity of the mirror system as a test for integrability. In fact, we will prove in [10] that a differential equation passes the Painlevé test (in the most strict sense) if and only if it has a regular mirror system. The second application is to use the mirror system to give a conceptual proof that the Laurent series generated by a successful application of the Painlevé test are indeed convergent. The argument is based on applying the Cauchy-Kowalevski theorem to the mirror system. In particular, such an argument always works for integrable ordinary differential equations. For integrable partial differential equations, however, the mirror system does not in general are in the form for which we can directly apply the Cauchy-Kowalevski theorem. In some cases, such as Burger's equation and the KdV equation, we may get around this by some further modifications. As a consequence, we are still able to give a conceptual proof of the convergence of the Laurent series from the Painlevé test to these equations.

In order to give a conceptual proof of the convergence of the Laurent series from the Painlevé test for other pertial differential equations, we will also propose an alternative algorithm. Despite complicated outcome of this algorithm, we can always apply the Cauchy-Kowalevski theorem at the end. As a result, we are able to give a conceptual proof of the convergence of the Laurent series from the Painlevé test to equations such as mKdV, cKdV, Boussinesq, KP, Sine-Gordon, etc. We also believe that successfully carrying out the alternative algorithm is equivalent to passing the Painlevé test.

This alternative algorithm was inspired by an example of Kruskal. In January of 1998, Kruskal discovered that if $u$ satisfies the second Painlevé equation, qthen $u^{-1}$ satisfies a 6 -th order regular analytic equation. Then the first author found that if $u$ satisfies the first Painlevé equation, then $u^{-\frac{1}{2}}$ satisfies an 8-th order regular analytic equation. Further experimentation led to the alternative algorithm. 
The paper is organized as follows: In Section 2, we present the algorithm for finding the mirror systems, using Burger's equation and the KdV equation as examples. In Section 3, we explain the relation between our algorithm and the Painlevé test. Then in Section 4, we show how to use the mirror system to test the integrability, using the generalized second Painlevé equation and the generalized KdV equation as examples. In Section 5, we explain how to use the mirror system to prove the convergence of the series obtained from the Painlevé test. Finally, we describe an alternative algorithm in Section 6.

2. Algorithm. In this section, we propose an algorithm for finding a regular system from a given (presumably integrable) differential equation. We consider a complex differential equation of the form

$$
\frac{\partial^{n} u}{\partial x^{n}}=F\left(x, z, u, \cdots, D^{\alpha^{*}} u\right)
$$

where

1. $u=u(x, z), x \in \mathbf{C}, z \in \mathbf{C}^{d}$;

2. The multi-indices $\alpha^{*}$ satisfy $\left|\alpha^{*}\right| \leq n$ and $\alpha^{*} \neq(n, 0, \cdots, 0)$;

3. $F$ is analytic in $x, z$, rational in $u$, and polynomial in derivatives of $u$.

$\underline{\text { Step } 1}$ Find the dominant behavior.

We look for a solution of the form $u(x, z) \sim u_{0}(x, z) \phi(x, z)^{k}$ near a non-characteristic, movable singularity manifold $\phi=0$ (by non-characteristic we have $\phi_{x} \neq 0$ along the singularity manifold). By solving the dominant equation we determine all possible $k$ 's. As in the Painlevé test, we require that all these $k$ 's are integers.

For example, consider Burger's equation

$$
u_{x x}=-u_{t}-u u_{x} .
$$

The substitution $u \sim u_{0} \phi^{k}$ into the equation tells us that the dominant equation is $u_{0} k(k-1) \phi^{k-2} \phi_{x}^{2} \sim-u_{0}^{2} k \phi^{2 k-1} \phi_{x}$. This implies $k-2=2 k-1$, or $k=-1$.

Step 2 Introduce indicial normalization.

For each of the $k$ 's found in the first step, we introduce the transform

$$
u=\theta^{k} \text {. }
$$

Since $u$ has order $k$ near its movable singularity, the new variable $\theta$ has order 1 . Therefore we call the transform (2.3) the indicial normalization.

Substituting (2.3) into (2.1), we get an equation for $\theta$. Near the non-characteristic, movable singularity manifold $\phi=0$, the equation is of the form

$$
\theta^{l} \frac{\partial^{n} \theta}{\partial x^{n}}=G\left(x, z, \theta, \cdots, D^{\alpha^{*}} \theta\right)
$$

where $G$ is analytic in all variables except $x$ and $z$, so the only singularities in $G$ are fixed ones.

For Burger's equation, we only need to consider the transform

$$
u=\theta^{-1} \text {. }
$$


After simple calculations, we get

$$
\theta \theta_{x^{2}}=-\theta \theta_{t}-\theta_{x}+2 \theta_{x}^{2}
$$

Note that this equation is singular when $\theta=0$.

$\underline{\text { Step } 3}$ Find leading behaviors for the derivatives of $\theta$ and the resonace derivatives.

We apply various derivatives to (2.4) and get a set of differential equations, which we denote by $\mathcal{E}=0$. From this set of equations, our ultimate goal is to find expansions

$$
D^{\alpha} \theta=\lambda_{\alpha}^{(0)}+\theta \lambda_{\alpha}^{(1)}+\theta^{2} \lambda_{\alpha}^{(2)}+\cdots,
$$

where $\lambda_{\alpha}^{(*)}$ are functions of $\theta_{z^{\beta}}$ and $\theta_{x^{j+1}} z^{\beta}$, for resonance derivatives $\theta_{x^{j+1}}$ (this notion will be introduced below). In this step, we only try to find the leading behaviors $\lambda_{\alpha}^{(0)}$.

We symbolically expand $\mathcal{E}$ in terms of $\theta: \mathcal{E}=\mathcal{E}_{0}+\theta \mathcal{E}_{1}+\theta^{2} \mathcal{E}_{2}+\cdots$, where each $\mathcal{E}_{i}$ is analytic in the derivatives of $\theta$. Then we try to solve $\mathcal{E}_{0}=0$ and find $\lambda_{\alpha}^{(0)}$.

Let us consider the example of Burger's equation. We apply $D^{\alpha}$, with $|\alpha| \leq 2$, to (2.6) and get the following degree $\leq 2$ part of $\mathcal{E}=0$

$$
\begin{aligned}
-\theta_{x}+2 \theta_{x}^{2}+\theta\left(-\theta_{t}-\theta_{x^{2}}\right) & =0, \\
-\theta_{t} \theta_{x}-\theta_{x^{2}}+3 \theta_{x} \theta_{x^{2}}+\theta\left(-\theta_{x t}-\theta_{x^{3}}\right) & =0, \\
-\theta_{t}^{2}-\theta_{x t}+4 \theta_{x} \theta_{x t}-\theta_{t} \theta_{x^{2}}+\theta\left(-\theta_{t^{2}}-\theta_{x^{2} t}\right) & =0, \\
-2 \theta_{x} \theta_{x t}-\theta_{t} \theta_{x^{2}}+3 \theta_{x^{2}}^{2}-\theta_{x^{3}}+2 \theta_{x} \theta_{x^{3}}+\theta\left(-\theta_{x^{2} t}-\theta_{x^{4}}\right) & =0, \\
-\theta_{t^{2}} \theta_{x}-2 \theta_{t} \theta_{x t}+3 \theta_{x t} \theta_{x^{2}}-\theta_{x^{2} t}+3 \theta_{x} \theta_{x^{2} t}-\theta_{t} \theta_{x^{3}} & \\
+\theta\left(-\theta_{x t^{2}}-\theta_{x^{3} t}\right) & =0, \\
-3 \theta_{t} \theta_{t^{2}}+4 \theta_{x t}^{2}-\theta_{x t^{2}}+4 \theta_{x} \theta_{x t^{2}}-\theta_{t^{2}} \theta_{x^{2}}-2 \theta_{t} \theta_{x^{2} t} & \\
+\theta\left(-\theta_{t^{3}}-\theta_{x^{2} t^{2}}\right) & =0 .
\end{aligned}
$$

By ignoring the terms containing $\theta$, we get the degree $\leq 2$ part of $\mathcal{E}_{0}=0$.

$$
\begin{aligned}
-\theta_{x}+2 \theta_{x}^{2} & =0, \\
-\theta_{t} \theta_{x}-\theta_{x^{2}}+3 \theta_{x} \theta_{x^{2}} & =0, \\
-\theta_{t}^{2}-\theta_{x t}+4 \theta_{x} \theta_{x t}-\theta_{t} \theta_{x^{2}} & =0, \\
-2 \theta_{x} \theta_{x t}-\theta_{t} \theta_{x^{2}}+3 \theta_{x^{2}}^{2}-\theta_{x^{3}}+2 \theta_{x} \theta_{x^{3}} & =0, \\
-\theta_{t^{2}} \theta_{x}-2 \theta_{t} \theta_{x t}+3 \theta_{x t} \theta_{x^{2}}-\theta_{x^{2} t}+3 \theta_{x} \theta_{x^{2} t}-\theta_{t} \theta_{x^{3}} & =0, \\
-3 \theta_{t} \theta_{t^{2}}+4 \theta_{x t}^{2}-\theta_{x t^{2}}+4 \theta_{x} \theta_{x t^{2}}-\theta_{t^{2}} \theta_{x^{2}}-2 \theta_{t} \theta_{x^{2} t} & =0 .
\end{aligned}
$$

By the non-characteristic assumption, we have $\theta_{x} \neq 0$ near the singularity $\phi=0$. Thus the solution of the equation (2.14) gives

$$
\theta_{x}=\frac{1}{2}+O(\theta)
$$

Substituting this into (2.15) and (2.16) and then solve for $\theta_{x^{2}}$ and $\theta_{x t}$, we have

$$
\begin{aligned}
& \theta_{x^{2}}=\theta_{t}+O(\theta), \\
& \theta_{x t}=2 \theta_{t}^{2}+O(\theta) .
\end{aligned}
$$


We further substitute (2.20), (2.21), and (2.22) into (2.17), (2.18), and (2.19). Then we find that (2.11) only yields a trivial relation

$$
0=O(\theta)
$$

while we can still find the leading behaviors of $\theta_{x^{2} t}$ and $\theta_{x t^{2}}$ from the other two equations

$$
\begin{aligned}
& \theta_{x^{2} t}=-4 \theta_{t}^{3}+\theta_{t^{2}}+2 \theta_{t} \theta_{x^{3}}+O(\theta), \\
& \theta_{x t^{2}}=-24 \theta_{t}^{4}+6 \theta_{t} \theta_{t^{2}}+4 \theta_{t}^{2} \theta_{x^{3}}+O(\theta) .
\end{aligned}
$$

The fact that (2.11) becomes trivial is related to the fact that in applying the Painlevé test, the Burger's equation has a resonance at $j=2$. In general, a resonance at $j$ in the Painlevé test corresponds to the fact that the leading behavior of $\theta_{x^{j+1}}$ can be arbitrarily chosen. Thus we call such $\theta_{x^{j+1}}$ a resonance derivative. For more detailed discussion about the correspondence, see Section 3.1.

As in the case of the Painlevé test, for an $n$-th order equation, we require that there are $(n-1)$ derivatives $\theta_{x^{j_{1}+1}}, \cdots, \theta_{x^{j_{n-1}+1}}, j_{1}<\cdots<j_{n-1}$, such that the leading behaviors cannot be determined. We also note that if the leading behavior of $\theta_{x^{j+1}}$ cannot be determined, then the leading behavior of $\theta_{x^{j+1} z^{\beta}}$ cannot be determined either. Thus we should not count $\theta_{x^{j+1}} z^{\beta}$ as independent resonnce derivatives.

If we cannot find enough resonance derivatives, then we cannot carry our algorithm further. In fact, this corresponds to failing the Painlevé test. Therefore from now on, we assume we can find enough resonance derivatives. Once we find all of them, we may stop finding expansions of more derivatives of $\theta$.

For the Burger's equation, we have already found the only resonance derivative $\theta_{x^{3}}$. Thus we have completed the third step.

Step 4 Find expansions for the (non-resonance) derivatives of $\theta$.

After finding the leading behaviors $\lambda_{\alpha}^{(0)}$, we try to find the first order behaviors $\lambda_{\alpha}^{(1)}$. This can be done by substituting $D^{\alpha} \theta=\lambda_{\alpha}^{(0)}+\theta \lambda_{\alpha}^{(1)}$ into $\mathcal{E}_{0}+\theta \mathcal{E}_{1}=0$ and solve for $\lambda_{\alpha}^{(1)}$.

Let us again consider the Burger's equation, for which the degree $\leq 2$ part of $\mathcal{E}_{0}+\theta \mathcal{E}_{1}=0$ is exactly $(2.8-2.13)$. However, we should drop (2.17) from the list because of the resoance derivative $\theta_{x^{3}}$.

We substitute $\theta_{x}=\frac{1}{2}+\theta \lambda_{1,0}^{(1)}$ and (2.21) into (2.8) to get $\lambda_{1,0}^{(1)}=2 \theta_{t}$. Thus

$$
\theta_{x}=\frac{1}{2}+2 \theta \theta_{t}+O\left(\theta^{2}\right)
$$

Similarly, we substitute $\theta_{x^{2}}=\theta_{t}+\theta \lambda_{2,0}^{(1)}, \theta_{x t}=2 \theta_{t}^{2}+\theta \lambda_{1,1}^{(1)}$ and (2.21), (2.22), (2.25) to $(2.11),(2.12)$, respectively. Solving for $\lambda_{2,0}^{(1)}$ and $\lambda_{1,1}^{(1)}$, we get

$$
\begin{aligned}
& \theta_{x^{2}}=\theta_{t}+\theta\left(-4 \theta_{t}^{2}+2 \theta_{x^{3}}\right)+O\left(\theta^{2}\right), \\
& \theta_{x t}=2 \theta_{t}^{2}+\theta\left(-24 \theta_{t}^{3}+2 \theta_{t^{2}}+4 \theta_{t} \theta_{x^{3}}\right)+O\left(\theta^{2}\right) .
\end{aligned}
$$

In general, further refinements of the expansions for the derivatives of $\theta$ are needed. Thus, after finding the leading and the first order behaviors, we substitute $D^{\alpha} \theta=\lambda_{\alpha}^{(0)}+\theta \lambda_{\alpha}^{(1)}+\theta^{2} \lambda_{\alpha}^{(2)}$ into $\mathcal{E}_{0}+\theta \mathcal{E}_{1}+\theta^{2} \mathcal{E}_{2}=0$ and solve for the second 
order behaviors. We should keep continuing until the highest resonance derivative appears in the expansions of $\theta_{x}, \cdots, \theta_{x^{n-1}}$.

For the Burger's equation, one more round of computation gives us

$$
\theta_{x}=\frac{1}{2}+2 \theta \theta_{t}+\theta^{2}\left(-12 \theta_{t}^{2}+2 \theta_{x^{3}}\right)+O\left(\theta^{3}\right) .
$$

which contains the only resonance derivative $\theta_{x^{3}}$. This completes the fourth step for the Burger's equation.

$\underline{\text { Step } 5}$ Introduce new variables for the mirror system.

The new variables are introduced by cutting at the expansions of $\theta_{x}, \cdots, \theta_{x^{n-1}}$ at the resonance derivatives. For the Burger's equation, we follow (2.28) and introduce $\xi$ by

$$
\theta_{x}=\frac{1}{2}+2 \theta \theta_{t}+\theta^{2} \xi
$$

The example of the Burger's equation is too simple to address a technical point. To illustrate this, we consider the example of the KdV equation

$$
u_{t}-6\left(u^{2}\right)_{x}+u_{x x x}=0 .
$$

The indicial normalization for the equation is $u=\theta^{-2}$. After carrying out the steps 1 through 4, we get two branches of expansions, one of which is

$$
\begin{aligned}
\theta_{x}= & 1-\theta^{2} \frac{\theta_{t}}{8}+\theta^{4}\left(-\frac{5 \theta_{t}^{2}}{192}+\frac{\theta_{x^{5}}}{24}\right) \\
& +\theta^{5} \frac{31 \theta_{t^{2}}}{192}+\theta^{6}\left(\frac{143 \theta_{t}^{3}}{4608}+\frac{41 \theta_{t} \theta_{x^{5}}}{2880}-\frac{7 \theta_{x^{7}}}{360}\right)+O\left(\theta^{7}\right), \\
\theta_{x^{2}}= & -\theta \frac{\theta_{t}}{4}+\theta^{3}\left(-\frac{\theta_{t}^{2}}{24}+\frac{\theta_{x^{5}}}{6}\right) \\
& +\theta^{4} \frac{59 \theta_{t^{2}}}{96}+\theta^{5}\left(\frac{107 \theta_{t}^{3}}{768}+\frac{\theta_{t} \theta_{x^{5}}}{30}-\frac{3 \theta_{x^{7}}}{40}\right)+O\left(\theta^{6}\right),
\end{aligned}
$$

where $\theta_{x^{5}}$ and $\theta_{x^{7}}$ are the resonance derivatives.

By cutting the expansion (2.30) at the first resonance $\theta_{x^{5}}$, we introduce a new variable $\xi$ by

$$
\theta_{x}=1-\theta^{2} \frac{\theta_{t}}{8}+\theta^{4} \xi
$$

The new variable $\xi$ and the resonance derivative $\theta_{x^{5}}$ are related by

$$
\xi=-\frac{5 \theta_{t}^{2}}{192}+\frac{\theta_{x^{5}}}{24}+\theta \frac{31 \theta_{t^{2}}}{192}+\theta^{2}\left(\frac{143 \theta_{t}^{3}}{4608}+\frac{41 \theta_{t} \theta_{x^{5}}}{2880}-\frac{7 \theta_{x^{7}}}{360}\right)+O\left(\theta^{3}\right) .
$$

We may formally converting this and get

$$
\theta_{x^{5}}=24 \xi+\frac{5 \theta_{t}^{2}}{8}-\theta \frac{31 \theta_{t^{2}}}{8}+\theta^{2}\left(-\frac{41 \xi \theta_{t}}{5}-\frac{23 \theta_{t}^{3}}{24}+\frac{7 \theta_{x^{7}}}{15}\right)+O\left(\theta^{3}\right) .
$$


Substituting this into the expansion (2.31), we have

$$
\theta_{x^{2}}=-\theta \frac{\theta_{t}}{4}+\theta^{3}\left(4 \xi+\frac{\theta_{t}^{2}}{16}\right)-\theta^{4} \frac{\theta_{t^{2}}}{32}+\theta^{5}\left(-\frac{17 \xi \theta_{t}}{30}+\frac{\theta_{t}^{3}}{2304}+\frac{\theta_{x^{7}}}{360}\right)+O\left(\theta^{6}\right) .
$$

By cutting the expansion above at the second resonance derivative $\theta_{x^{7}}$, we get

$$
\theta_{x^{2}}=-\theta \frac{\theta_{t}}{4}+\theta^{3}\left(4 \xi+\frac{\theta_{t}^{2}}{16}\right)-\theta^{4} \frac{\theta_{t^{2}}}{32}+\theta^{5} \eta
$$

which introduces another new variable $\eta$.

Note that the new variables are in some sense equivalent to the resonance derivatives. The key technical point is that after introducing a new variable ( $\xi$, for $\mathrm{KdV}$ ), we should express the corresponding resonance derivative $\left(\theta_{x^{5}}\right)$ in terms of the new variable and the other new variables introduced before. Then we need to substitute the expression to update the expansions for higher order derivatives $\left(\theta_{x^{2}}\right)$, in which the lower order resonance derivatives $\left(\theta_{x^{5}}\right)$ have all been replaced by the new variables introduced so far $(\xi)$.

$\underline{\text { Step } 6}$ Derive the mirror system.

After completing the last step, we introduce new variables $\xi_{1}, \cdots, \xi_{n-1}$ by

$$
\left\{\begin{aligned}
\theta_{x} & =\bar{\lambda}_{1}^{(0)}+\theta \bar{\lambda}_{1}^{(1)}+\cdots+\theta^{j_{1}-1} \bar{\lambda}_{1}^{\left(j_{1}-1\right)}+\theta^{j_{1}} \xi_{1}, \\
\theta_{x^{2}} & =\bar{\lambda}_{2}^{(0)}+\theta \bar{\lambda}_{2}^{(1)}+\cdots+\theta^{j_{2}-2} \bar{\lambda}_{2}^{\left(j_{2}-2\right)}+\theta^{j_{2}-1} \xi_{2}, \\
& \vdots \\
\theta_{x^{n-1}} & =\bar{\lambda}_{n-1}^{(0)}+\theta \bar{\lambda}_{n-1}^{(1)}+\cdots+\theta^{j_{n-1}-n+2} \bar{\lambda}_{n-1}^{\left(j_{n-1}-n+2\right)}+\theta^{j_{n-1}-n+1} \xi_{n-1},
\end{aligned}\right.
$$

where $\bar{\lambda}_{q}^{(*)}$ are functions of $\theta_{z^{\beta}},\left(\xi_{1}\right)_{z^{\beta}}, \cdots,\left(\xi_{q-1}\right)_{z^{\beta}}$. The variables for our mirror system are $\theta, \xi_{1}, \cdots, \xi_{n-1}$. It remains to find the equations for their derivatives with respect to $x$.

From $(2.34)$ and $\left(\theta_{x}\right)_{x}=\theta_{x^{2}}$, we find

$$
\begin{aligned}
\theta^{j_{1}}\left(\xi_{1}\right)_{x}= & \bar{\lambda}_{2}^{(0)}+\theta \bar{\lambda}_{2}^{(1)}+\cdots+\theta^{j_{2}-2} \bar{\lambda}_{2}^{\left(j_{2}-2\right)}+\theta^{j_{2}-1} \xi_{2} \\
& -D_{x}\left[\bar{\lambda}_{1}^{(0)}+\theta \bar{\lambda}_{1}^{(1)}+\cdots+\theta^{j_{1}-1} \bar{\lambda}_{1}^{\left(j_{1}-1\right)}\right]-p_{1} \theta^{j_{1}-1} \theta_{x} \xi_{1} .
\end{aligned}
$$

The right side involves $\theta_{z^{\beta}}, \theta_{x z^{\beta}},\left(\xi_{1}\right)_{z^{\beta}}$, and $\xi_{2}$. We substitute $\theta_{x z^{\beta}}$ by the first formula in (2.34). Then the right side no longer involves derivatives in $x$. After simplification, we will see that the right side has a common factor $\theta^{j_{1}}$. Dividing the factor, we get an equation for $\left(\xi_{1}\right)_{x}$.

Similarly, from (2.34), $\left(\theta_{x^{2}}\right)_{x}=\theta_{x^{3}}$, and the equation for $\left(\xi_{1}\right)_{x}$ we just obtained, we may find the equation for $\left(\xi_{2}\right)_{x}$. We may continue one by one and find the equations for $\left(\xi_{1}\right)_{x}, \cdots,\left(\xi_{n-2}\right)_{x}$.

To find an equation for $\left(\xi_{n-1}\right)_{x}$, we substitute $(2.34)$ and their derivatives in $z$ into the right side of (2.4). We think of the left side of $(2.4)$ as $\theta^{l}\left(\theta_{x^{n-1}}\right)_{x}$ and replace $\theta_{x^{n-1}}$ by the the formula in (2.34). Then we get an equality of the form

$$
\theta^{l+j_{n-1}-n+1}\left(\xi_{n-1}\right)_{x}+\cdots=\cdots,
$$


where $\cdots$ on both sides only involve $\theta_{z^{\beta}},\left(\xi_{1}\right)_{z^{\beta}}, \cdots,\left(\xi_{n-1}\right)_{z^{\beta}},\left(\xi_{1}\right)_{x z^{\beta}}, \cdots,\left(\xi_{n-2}\right)_{x z^{\beta}}$. Then we substitute the known equations for $\left(\xi_{1}\right)_{x}, \cdots,\left(\xi_{n-2}\right)_{x}$ into the derivatives $\left(\xi_{1}\right)_{x z^{\beta}}, \cdots,\left(\xi_{n-2}\right)_{x z^{\beta}}$. The result is an expression of $\theta^{l+j_{n-1}-n+1}\left(\xi_{n-1}\right)_{x}$ in terms of $\theta_{z^{\beta}},\left(\xi_{1}\right)_{z^{\beta}}, \cdots,\left(\xi_{n-1}\right)_{z^{\beta}}$. After simplification and dividing by $\theta^{l+j_{n-1}-n+1}$, we will get a regular equation for $\left(\xi_{n-1}\right)_{x}$.

For example, we substitute (2.29) into (2.6) and get

$$
\theta\left(\frac{1}{2}+2 \theta \theta_{t}+\theta^{2} \xi\right)_{x}=-\theta \theta_{t}-\theta_{x}+2 \theta_{x}^{2}
$$

From (2.29), we have formulae for $\theta_{x}$ and $\theta_{x t}$. Substituting these into (2.35), we get an equation

$$
-2 \theta_{t} \xi-2 \theta \xi_{t}-4 \theta_{t^{2}}-\xi_{x}=0 .
$$

Combining (2.29) and (2.36) together, we have a differential system for $(\theta, \xi)$

$$
\left\{\begin{aligned}
\theta_{x} & =\frac{1}{2}+2 \theta \theta_{t}+\theta^{2} \xi \\
\xi_{x} & =-4 \theta_{t^{2}}-2 \theta_{t} \xi-2 \theta \xi_{t} .
\end{aligned}\right.
$$

This is a mirror system for Burger's equation.

As for the one branch of the KdV equation discussed in step 5, we identify (2.33) with the derivative of $(2.32)$ and solve for $\xi_{x}$. The result is

$$
\xi_{x}=-\frac{3 \theta_{t^{2}}}{64}+\theta\left(\eta+\frac{5 \xi \theta_{t}}{4}\right)+\theta^{2} \frac{\xi_{t}}{8}-4 \theta^{3} \xi^{2} .
$$

We substitute (2.32) and (2.33) into the equation

$$
-\theta^{2} \theta_{t}+12 \theta_{x}-12 \theta_{x}^{3}+9 \theta \theta_{x} \theta_{x^{2}}-\theta^{2} \theta_{x^{3}}=0
$$

obtained by applying the indicial normalization $u=\theta^{-2}$ to the $\mathrm{KdV}$ equation. Then we replace $\theta_{x^{3}}$ by the derivative of $(2.33)$ and solve for $\eta_{x}$. The result is

$$
\begin{aligned}
\eta_{x}= & -\frac{\xi_{t}}{4}+\frac{3 \theta_{t} \theta_{t^{2}}}{256}+\theta\left(4 \xi^{2}-\frac{\eta \theta_{t}}{2}-\frac{5 \xi \theta_{t}^{2}}{16}-\frac{\theta_{t^{3}}}{256}\right) \\
& +\theta^{2}\left(\frac{\theta_{t} \xi_{t}}{8}-\frac{\xi \theta_{t^{2}}}{32}\right)+\theta^{3}\left(4 \xi \eta+\frac{9 \xi^{2} \theta_{t}}{2}+\frac{\xi_{t^{2}}}{32}\right)-12 \theta^{5} \xi^{3} .
\end{aligned}
$$

The regular equations (2.32), (2.38) and (2.39) form a mirror system of the $\mathrm{KdV}$ equation.

3. Mirror System and Painlevé Test. In this section, we explain the relation between our algorithm and the Painlevé test from two perspectives. In fact, we have in [10] a theoretical argument that successfully carrying out our algorithm (and get a regular mirror system at the end) is equivalent to passing the Painlevé test.

3.1. Resonances and Resonace Derivatives. For a solution $u$ near a noncharacteristic, movable singularity manifold $\phi(x, z)=0$, the Painlevé test assumes the expansion

$$
u=\phi^{k} \sum_{i=0}^{\infty} u_{i} \phi^{i}
$$


Without loss of generality, we may further assume

$$
\phi=x+\psi(z), \quad u_{i}=u_{i}(z)
$$

by the non-characteristic assumption. Rewrite (2.3) formally as

$$
\theta=\phi\left(\sum_{i=0}^{\infty} u_{i} \phi^{i}\right)^{1 / k}
$$

Then for any positive integer $j$, we have

$$
\theta_{x^{j+1}} \sim \frac{(j+1) !}{k} u_{0}^{-1+\frac{1}{k}} u_{j}+\text { a function depending on } u_{i} \text { 's with } i<j,
$$

to the leading order of $\theta$. If $j$ is a resonance in the Painlevé test, then $u_{j}$ can be arbitrarily chosen (as a funciton of $z$ ). As a result, $\theta_{x^{j+1}}$ (and its derivatives in $z$ ) can be equally arbitrarily chosen. As we have seen in examples such as (2.11), this happens in our algorithm when the equation from $\mathcal{E}_{0}=0$ that should compute the leading behavior of $\theta_{x^{j+1}}$ for us becomes trivial.

3.2. Painlevé Revisited. As pointed out in the introduction, Painlevé already used the mirror system in his proof of the Painlevé property for

$$
y^{\prime \prime}=6 y^{2}+x .
$$

In this section, we analyze in detail what he actually did.

On page 228 of [18], Painlevé started by studying the generalized Laurent expansion of a solution $y$ at algebraic singular points $x_{0}$. By dominant balance, he found the leading term of this series. Then he made use of the equation to find the expansion

$$
y=\frac{1}{\left(x-x_{0}\right)^{2}}-\frac{x_{0}}{10}\left(x-x_{0}\right)^{2}-\frac{1}{6}\left(x-x_{0}\right)^{3}+h\left(x-x_{0}\right)^{4}+\frac{x_{0}^{2}}{18}\left(x-x_{0}\right)^{6}+\cdots,
$$

where $h$ is an arbitrary constant (resonance). Next he rewrote the expansion (3.4) into

$$
y=\frac{1}{\left(x-x_{0}\right)^{2}}-\frac{x}{10}\left(x-x_{0}\right)^{2}-\frac{1}{15}\left(x-x_{0}\right)^{3}+h\left(x-x_{0}\right)^{4}+\frac{x^{2}}{18}\left(x-x_{0}\right)^{6}+\cdots,
$$

so that the coefficients are all independent of the specific point $x_{0}$. Taking derivative of (3.5) yields

$$
y^{\prime}=-\frac{2}{\left(x-x_{0}\right)^{3}}-\frac{x}{5}\left(x-x_{0}\right)-\frac{3}{10}\left(x-x_{0}\right)^{2}+4 h\left(x-x_{0}\right)^{3}+\frac{x^{2}}{3}\left(x-x_{0}\right)^{5}+\cdots .
$$

Eliminating $\left(x-x_{0}\right)$ from (3.5) and (3.6), he got the expansion

$$
y^{\prime}=-\frac{2}{\epsilon y^{3 / 2}}-\frac{x \epsilon y^{1 / 2}}{2}-\frac{y}{2}+7 h \epsilon y^{3 / 2}+\cdots, \quad \epsilon= \pm 1
$$

In principle, one may invert (3.5) to get an expansion of $\left(x-x_{0}\right)$ in terms of $\epsilon y^{1 / 2}$ and then put the expansion into (3.6) to get (3.7).

A subtle point in Painlevé's process is that he converted the familiar series (3.4) that people would usually use for singularity analysis (as in the Painlevé test) to a less 
familiar series (3.6). In fact, Painlevé's intention was not to use the series to analyze the singularity. He merely made use of the series in order to derive the expansion (3.7) and ultimately his differential system. Therefore it is very important that the coefficients in the series should not depend on the point $x_{0}$, as is required by the system.

Next Painlevé introduced (the indicial normalization) $z=\epsilon y^{1 / 2}$ to make powers in the expansion (3.7) integral. Then he cut the expansion (3.7) at the arbitrary constant $h$ by introducing a new variable $u$. This gives rise to the transformation

$$
y=\frac{1}{z^{2}}, \quad y^{\prime}=-\frac{2}{z^{3}}-\frac{x z}{2}-\frac{z^{2}}{2}+u z^{3}
$$

that converts the original equation (3.3) into his regular system.

Note that eliminating $y$ from (3.8) gives us

$$
\frac{d z}{d x}=1+\frac{x z^{4}}{4}+\frac{z^{5}}{4}-\frac{u}{2} z^{6}
$$

This is exactly the change of variable one would get through our algorithm, except we would call $-\frac{1}{2} u$ the new variable. While Painlevé made concrete computations of the generalized Laurent series in order to introduce his new variable, our algorithm provides a more direct and systematic way of accomplishing the same.

4. Mirror System as a Detector of Integrability. In this section, we will show more example of the mirror system. In fact, to illustrate that our algorithm is equivalent to the Painlevé test, we will start with a generally non-integrable equation and derive all the integrable cases.

4.1. The Generalized Painlevé II. Consider the following generalization of the second Painlevé equation

$$
u^{\prime \prime}=2 u^{3}+a u+b,
$$

where $a=a(t)$ and $b=b(t)$ are analytic functions. From the dominant balance, we need to apply the indicial normalization $u=\theta^{-1}$. This converts the equation (4.1) to

$$
-2-a \theta^{2}-b \theta^{3}+2{\theta^{\prime}}^{2}-\theta \theta^{\prime \prime}=0 .
$$

Our algorithm leads to two possible branches of expansions, on of which is

$$
\begin{aligned}
\theta^{\prime}= & 1+\frac{a \theta^{2}}{2}+\theta^{3}\left(b+\frac{a^{\prime}}{2}\right) \\
& +\theta^{4}\left(-\frac{a^{2}}{6}+\frac{13 b^{\prime}}{12}+\frac{7 a^{\prime \prime}}{24}+\frac{\theta^{(5)}}{24}\right)+\cdots,
\end{aligned}
$$

where $\theta^{(5)}$ is the only resonance derivative. This suggests us to introduce a new variable $\xi$ (for this branch) by

$$
\theta^{\prime}=1+\frac{a \theta^{2}}{2}+\theta^{3}\left(b+\frac{a^{\prime}}{2}\right)+\theta^{4} \xi
$$


Substitute $\theta^{\prime \prime}$ in (4.2) by the derivative of (4.3). Then substitute $\theta^{\prime}$ in the resulting equation by (4.3). We have

$$
\begin{aligned}
\xi^{\prime}= & \left(-b^{\prime}-\frac{a^{\prime \prime}}{2}\right) \theta^{-1}+\left(-\frac{a b}{2}-\frac{a a^{\prime}}{4}\right)+\theta\left(-b^{2}-a \xi-b a^{\prime}-\frac{a^{2}}{4}\right) \\
& +\theta^{2}\left(-3 b \xi-\frac{3 \xi a^{\prime}}{2}\right)-2 \theta^{3} \xi^{2} .
\end{aligned}
$$

The equations (4.3) and (4.4) form a system for $(\theta, \xi)$. The system is regular at $\theta=0$ if and only if

$$
-b^{\prime}-\frac{a^{\prime \prime}}{2}=0
$$

Similarly, for the other branch of expansions, we should introduce a new variable $\xi$ by

$$
\theta^{\prime}=-1-\frac{a \theta^{2}}{2}+\theta^{3}\left(-b+\frac{a^{\prime}}{2}\right)+\theta^{4} \xi .
$$

Then the equation (4.2) is converted to

$$
\begin{aligned}
\xi^{\prime}= & \left(b^{\prime}-\frac{a^{\prime \prime}}{2}\right) \theta^{-1}+\left(-\frac{a b}{2}+\frac{a a^{\prime}}{4}\right)+\theta\left(-b^{2}+a \xi+b a^{\prime}-\frac{a^{\prime 2}}{4}\right) \\
& +\theta^{2}\left(3 b \xi-\frac{3 \xi a^{\prime}}{2}\right)-2 \theta^{3} \xi^{2} .
\end{aligned}
$$

Again, the system (4.6) and (4.7) for $(\theta, \xi)$ is regular if and only if

$$
b^{\prime}-\frac{a^{\prime \prime}}{2}=0
$$

In general, both branches of expansions may appear. Therefore, in order that (4.1) to be integrable, we expect that the mirror systems for both branches should be regular. In other words, we need both (4.5) and (4.8) hold. This leads to

$$
a^{\prime \prime}=b^{\prime}=0 \text {. }
$$

After a linear change of variables, we end up with the standard second Painlevé equation

$$
u^{\prime \prime}=2 u^{3}+z u+\alpha
$$

4.2. The Generalized KdV. In section 7.2 .5 of [1], the Painlevé test was used to show that the generalized $\mathrm{KdV}$ equation

$$
u_{t}-6\left(u^{2}\right)_{x}+u_{x x x}+a(t) u=0,
$$

where $a(t)$ is an analytic function, is integrable if and only if

$$
a(t)=0 \quad \text { or } \quad a(t)=\frac{1}{2\left(t-t_{0}\right)} .
$$


The first case may be normalized to become the KdV equation

$$
u_{t}+6 u u_{x}+u_{x x x}=0 .
$$

The second case may be normalized to become the cylindrical KdV equation

$$
u_{t}+6 u u_{x}+u_{x x x}+\frac{u}{2 t}=0 .
$$

Now we try to use our algorithm to get the same conclusion. For the indicial normalization $u=\theta^{-2}$, we have the equation for $\theta$

$$
\theta^{3} \frac{a(t)}{2}-\theta^{2} \theta_{t}+12 \theta_{x}-12 \theta_{x}^{3}+9 \theta \theta_{x} \theta_{x^{2}}-\theta^{2} \theta_{x^{3}}=0
$$

Our algorithm leads to two possible branches of expansions. One is

$$
\begin{aligned}
\theta_{x}= & 1-\theta^{2} \frac{\theta_{t}}{8}+\theta^{3} \frac{a(t)}{6}+\theta^{4}\left(-\frac{5 \theta_{t}^{2}}{192}+\frac{\theta_{x^{5}}}{24}\right)+\theta^{5}\left(\frac{11 a(t) \theta_{t}}{32}+\frac{31 \theta_{t^{2}}}{192}\right) \\
& +\theta^{6}\left(-\frac{691 a(t)^{2}}{480}-\frac{169 a^{\prime}(t)}{240}+\frac{143 \theta_{t}^{3}}{4608}+\frac{41 \theta_{t} \theta_{x^{5}}}{2880}-\frac{7 \theta_{x^{7}}}{360}\right)+\cdots, \\
\theta_{x^{2}}= & -\theta \frac{\theta_{t}}{4}+\theta^{2} \frac{a(t)}{2}+\theta^{3}\left(-\frac{\theta_{t}^{2}}{24}+\frac{\theta_{x^{5}}}{6}\right)+\theta^{4}\left(\frac{19 a(t) \theta_{t}}{16}+\frac{59 \theta_{t^{2}}}{96}\right) \\
& +\theta^{5}\left(-\frac{1313 a(t)^{2}}{240}-\frac{649 a^{\prime}(t)}{240}+\frac{107 \theta_{t}^{3}}{768}+\frac{\theta_{t} \theta_{x^{5}}}{30}-\frac{3 \theta_{x^{7}}}{40}\right)+\cdots
\end{aligned}
$$

This leads to the introduction of new variables $\xi$ and $\eta$ by

$$
\begin{aligned}
\theta_{x} & =1-\theta^{2} \frac{\theta_{t}}{8}+\theta^{3} \frac{a(t)}{6}+\theta^{4} \xi \\
\theta_{x^{2}} & =-\theta \frac{\theta_{t}}{4}+\theta^{2} \frac{a(t)}{2}+\theta^{3}\left(4 \xi+\frac{\theta_{t}^{2}}{16}\right)+\theta^{4}\left(-\frac{3 a(t) \theta_{t}}{16}-\frac{\theta_{t^{2}}}{32}\right)+\theta^{5} \eta .
\end{aligned}
$$

If (4.9) is integrable, then we expect the mirror system for $(\theta, \xi, \eta)$ to be regular.

As in the case of the KdV equation, the change of variables (4.11) and (4.12) from $\left(\theta, \theta_{x}, \theta_{x^{2}}\right)$ to $(\theta, \xi, \eta)$ converts $(4.10)$ to

$$
\begin{aligned}
\xi_{x}= & -\frac{a(t) \theta_{t}}{48}-\frac{3 \theta_{t^{2}}}{64}+\theta\left(-\frac{a(t)^{2}}{12}+\eta+\frac{a^{\prime}(t)}{48}+\frac{5 \xi \theta_{t}}{4}\right) \\
& +\theta^{2}\left(-\frac{7 a(t) \xi}{6}+\frac{\xi_{t}}{8}\right)-4 \theta^{3} \xi^{2}, \\
\eta_{x}= & \theta^{-1}\left(-\frac{a(t)^{2}}{12}-\frac{a^{\prime}(t)}{24}\right)+\frac{a(t) \xi}{6}+\frac{a(t) \theta_{t}^{2}}{128}-\frac{\xi_{t}}{4}+\frac{3 \theta_{t} \theta_{t^{2}}}{256} \\
& +\theta\left(4 \xi^{2}+\frac{a(t)^{2} \theta_{t}}{16}-\frac{\eta \theta_{t}}{2}+\frac{a^{\prime}(t) \theta_{t}}{96}-\frac{5 \xi \theta_{t}^{2}}{16}-\frac{13 a(t) \theta_{t^{2}}}{384}-\frac{\theta_{t^{3}}}{256}\right) \\
& +\theta^{2}\left(-\frac{a(t)^{3}}{18}+\frac{2 a(t) \eta}{3}+\frac{a(t) a^{\prime}(t)}{32}+\frac{a^{\prime \prime}(t)}{192}+\frac{21 a(t) \xi \theta_{t}}{16}+\frac{\theta_{t} \xi_{t}}{8}-\frac{\xi \theta_{t^{2}}}{32}\right) \\
& +\theta^{3}\left(-a(t)^{2} \xi+4 \xi \eta+\frac{9 \xi^{2} \theta_{t}}{2}+\frac{3 a(t) \xi_{t}}{16}+\frac{\xi_{t^{2}}}{32}\right) \\
& -6 a(t) \theta^{4} \xi^{2}-12 \theta^{5} \xi^{3} .
\end{aligned}
$$


Thus, in order for the mirror system (consisting of (4.11), (4.13) and (4.14)) to be regular near $\theta=0$, the function $a(t)$ must satisfy

$$
-\frac{a(t)^{2}}{12}-\frac{a^{\prime}(t)}{24}=0 .
$$

The solution of this equation gives either

$$
a(t)=0, \quad \text { or } \quad a(t)=\frac{1}{2\left(t-t_{0}\right)} .
$$

For the other branch, we introduce

$$
\begin{aligned}
\theta_{x} & =-1-\theta^{2} \frac{\theta_{t}}{8}+\theta^{3} \frac{a(t)}{6}+\theta^{4} \xi \\
\theta_{x^{2}} & =\theta \frac{\theta_{t}}{4}-\theta^{2} \frac{a(t)}{2}+\theta^{3}\left(-4 \xi+\frac{\theta_{t}^{2}}{16}\right)+\theta^{4}\left(-\frac{3 a(t) \theta_{t}}{16}-\frac{\theta_{t^{2}}}{32}\right)+\theta^{5} \eta .
\end{aligned}
$$

We can also obtain a mirror system for these new variables $(\theta, \xi, \eta)$. Again, the system becomes regular if and only if equation (4.15) holds.

5. Convergence of Series in Painlevé Test. The Painlevé test produces the following formal solution of Burger's equation near movable singularity $x=\psi(t)$

$$
u=\frac{2}{x-\psi(t)}+\psi^{\prime}(t)+r(t)(x-\psi(t))+\cdots,
$$

where $r(t)$ (the only resonance) is an arbitrary function. The solution is formal in the sense that the Painlevé test cannot tell us the convergence of the series.

By converting Burger's equation into an integral equation and applying contractionmapping argument, Joshi and Petersen [13] have shown that the series is actually convergent to a solution of Burger's equation for any given holomorphic functions $\psi(t)$ and $r(t)$. Here we prove the same result by making use of the mirror system and the Cauchy-Kowalevski theorem in place of the integral equation and the contractionmapping theorem.

The mirror system (2.37) for the Burger's equation is not suitable for applying the Cauchy-Kowalevski theorem, because of the second order derivative $\theta_{t^{2}}$ on the right side. By introducing a new variable $\alpha=\theta_{t}$, we may extend the mirror system to

$$
\left\{\begin{aligned}
\theta_{x} & =\frac{1}{2}+2 \theta \alpha+\theta^{2} \xi \\
\xi_{x} & =-4 \alpha_{t}-2 \alpha \xi-2 \theta \xi_{t} \\
\alpha_{x} & =2 \alpha^{2}+2 \alpha \theta \xi+2 \theta \alpha_{t}+\theta^{2} \xi_{t}
\end{aligned}\right.
$$

where we use $\alpha_{x}=\left(\theta_{x}\right)_{t}$ to find the third equation. The extended mirror system (5.2) is suitable for applying the Cauchy-Kowalevski theorem.

The next thing we need to do is to convert the series (5.1) into an (equivalent) initial value condition for (5.2) along the singularity manifold $x=\psi(t)$. From (5.1) and $\theta=u^{-1}$, we have

$$
\theta=-\frac{1}{2}(x-\psi)-\frac{\psi^{\prime}}{4}(x-\psi)^{2}+\left(\frac{\psi^{\prime 2}}{8}-\frac{r}{4}\right)(x-\psi)^{3}+\cdots
$$


Taking derivative of this with respect to $t$, we have

$$
\alpha=-\frac{\psi^{\prime}}{2}+\frac{\psi^{\prime 2}}{2}(x-\psi)+\left(\frac{3 r \psi^{\prime}}{4}-\frac{3 \psi^{\prime 3}}{8}-\frac{\psi^{\prime \prime}}{4}\right)(x-\psi)^{2}+\cdots
$$

Substituting the series for $\theta$ and $\alpha$ into the first equation in (5.2) and solve for $\xi$, we have

$$
\xi=-3 r-\frac{3 \psi^{\prime 2}}{2}+\cdots
$$

From these power series, we find the following initial data for the mirror system along the singularity

$$
\theta=0, \quad \xi=-3 r(t)-\frac{3 \psi^{\prime}(t)^{2}}{2}, \quad \alpha=-\frac{\psi^{\prime}(t)}{2}, \quad \text { at } x=\psi(t) .
$$

Now we are ready to show the convergence of (5.1). By the Cauchy-Kowalevski theorem, the extended mirror system (5.2) with the initial value condition (5.4) has a unique analytic solution $(\theta(x, t), \alpha(x, t), \xi(x, t))$ near $x=\psi(t)$. Then $u=\theta^{-1}$ is a solution of Burger's equation near $x=\psi(t)$. Moreover, from the usual power series method, we find the expansion for $\theta$ is indeed (5.3). Then an easy computation shows that the Laurent series of $u=\theta^{-1}$ is exactly (5.1).

Our proof of the convergence is a routine one:

1. Use our algorithm to find the mirror system and, if necessary, extend the system so that the Cauchy-Kowalevski theorem is applicable;

2. Convert the given resonance functions into an initial condition for the (extended) mirror system;

3. Use power series method to solve the initial value problem (only up to certain power so that all resonance functions appear);

4. Verify that the inverse indicial normalization converts the power series obtained from the mirror system into the Laurent series one expects from the Painlevé test.

We note that for integrable ordinary differential equations, the right side of the mirror system involves no derivatives. Therefore the Cauchy-Kowalevski theorem is always applicable. As a result, we always have a routine conceptual proof of the convergence of the series from the Painlevé test. Our paper [10] contains more comprehensive discussion on this point.

Let us illustrate the routine again with the KdV equation. The formal Laurent series near movable singularity $x=\psi(t)$ produced by the Painlevé test is

$$
u=(x-\psi)^{-2}-\frac{\psi^{\prime}}{12}+r_{1}(x-\psi)^{2}-\frac{\psi^{\prime \prime}}{72}(x-\psi)^{3}+r_{2}(x-\psi)^{4}+\cdots,
$$

where $r_{1}=r_{1}(t)$ and $r_{2}=r_{2}(t)$ are arbitrary functions. Joshi and Srinivasan [14] showed that the series must converge. Using our method, we extend the mirror system (2.32), (2.38), (2.39) by introducing new variables $\alpha=\theta_{t}, \beta=\theta_{t^{2}}$, and $\gamma=\xi_{t}$. The extended system for $(\theta, \xi, \eta, \alpha, \beta, \gamma)$ consists of their first order derivatives in $x$ on the left side, and analytic functions of the six variables and their first order derivatives in $t$ on the right side. Therefore the Cauchy-Kowalevski theorem can be applied to the extended system. 
Then we need to convert the series (5.5) into the following initial data for the extended mirror system at $x=\psi(t)$ :

$$
\left\{\begin{aligned}
\theta & =0 \\
\xi & =-\frac{5 r_{1}(t)}{2}-\frac{5 \psi^{\prime}(t)^{2}}{384} \\
\eta & =-7 r_{2}(t)-\frac{55 r_{1}(t) \psi^{\prime}(t)}{24}-\frac{73 \psi^{\prime}(t)^{3}}{13824} \\
\alpha & =-\psi^{\prime}(t) \\
\beta & =-\psi^{\prime \prime}(t) \\
\gamma & =-\frac{5 r_{1}^{\prime}(t)}{2}-\frac{7 \psi^{\prime}(t) \psi^{\prime \prime}(t)}{96}
\end{aligned}\right.
$$

Then we find the power series solution of the initial value problem. The power expansion for $\theta$ is

$$
\begin{aligned}
\theta= & (x-\psi(t))+\frac{\psi^{\prime}(t)}{24}(x-\psi(t))^{3}+\left(\frac{\psi^{\prime}(t)^{2}}{384}-\frac{r_{1}(t)}{2}\right)(x-\psi(t))^{5} \\
& +\frac{\psi^{\prime \prime}(t)}{144}(x-\psi(t))^{6}+\left(\frac{5 \psi^{\prime}(t)^{3}}{27648}-\frac{r_{1}(t) \psi^{\prime}(t)}{16}-\frac{r_{2}(t)}{2}\right)(x-\psi(t))^{7}+\cdots
\end{aligned}
$$

It is then not difficult to verify that $u=\theta^{-2}$ has expansion (5.5).

Finally, we remark that all the other results in [12] [13] [14], such as well-posedness of the WTC Cauchy problem, will follow from the similar theorems on the ordinary Cauchy problems.

6. An Alternative Algorithm. In applying the mirror system to prove the convergence of the formal Laurent series solutions obtained from the PDE Painlevé test, we encounter the problem of applying the Cauchy-Kowalevski theorem. While we may use a little trick to get around the problem for Burger's equation and KdV equation, the trick does not always work. The Boussinesq equation is one such example.

In this section, we provide another algorithm that gives rise to a regular equation for which we can always apply the Cauchy-Kowalevski theorem. As a consequence, we can also give a conceptual proof of the convergence of the formal Laurent series solutions for many other integrable PDEs, including the Boussinesq equation.

In the alternative algorithm, we follow the first four steps in the algorithm in Section 2, with additional attension paid to the following:

1. Keep track of the derivatives involved in the remainders of expansions;

2. Find "compatibility relations" corresponding to resonance derivatives;

3. Find expansions (and compatibility relations) for all derivatives up to the largest order resonance derivative.

Let us explain these through the examples of the Burger's equation and the $\mathrm{KdV}$ equation.

We denote by $O_{A, m}\left(\theta^{l}\right)$ an expression of the form $\theta^{l} R\left(x, z, \cdots, D^{\beta} \theta\right)$, where $0 \leq$ $|\beta| \leq m$, and $R$ is analytic near $\theta=0$. Then for the Burger's equation, the equation (2.14) should really be more accurately written as $-\theta_{x}+2 \theta_{x}^{2}=O_{A, 2}(\theta)$, and the solution (2.20) is better described as

$$
\theta_{x}=\frac{1}{2}+O_{A, 2}(\theta)
$$


Similarly, all the expansions for the Burger's equation in Section 2 had remainders of the form $O_{A, \leq 4}(\theta)$ or $O_{A, \leq 4}\left(\theta^{2}\right)$.

Recall that $\theta_{x^{3}}$ was the only resonance derivative for the Burger's equation because the corresponding equation (2.17) that presumably computes the leading behavior became $0=O_{A, 4}(\theta)$ after substituting (2.20), (2.21), and (2.22) into the equation. However, if, in the second round of computation, we substitute (2.25), (2.26), and (2.27) into (2.11), then we get a nontrivial relation

$$
\theta\left(-3 \theta_{t^{2}}+8 \theta_{t} \theta_{x^{3}}-\theta_{x^{4}}\right)=O_{A, 4}\left(\theta^{2}\right) .
$$

Dividing $\theta$, we have

$$
-3 \theta_{t^{2}}+8 \theta_{t} \theta_{x^{3}}-\theta_{x^{4}}=O_{A, 4}(\theta) .
$$

This is the compatibility relation corresponding to the resonance derivative $\theta_{x^{3}}$.

After finishing the first four steps (and paying the additional attension), we are ready to construct a regular fifth order equation for $\theta$ for the Burger's equation. We we apply $D_{x^{3}}$ to $(2.6)$ to get

$$
\theta \theta_{x^{5}}=-3 \theta_{x t} \theta_{x^{2}}-3 \theta_{x} \theta_{x^{2} t}-\theta_{t} \theta_{x^{3}}+8 \theta_{x^{2}} \theta_{x^{3}}-\theta \theta_{x^{3} t}-\theta_{x^{4}}+\theta_{x} \theta_{x^{4}} .
$$

Substituting (2.20-2.24), keeping in mind of the remainders $O_{A, 4}\left(\theta^{2}\right)$, and simplifying, we have

$$
\theta \theta_{x^{5}}=\frac{-3 \theta_{t^{2}}}{2}+4 \theta_{t} \theta_{x^{3}}-\frac{\theta_{x^{4}}}{2}+O_{A, 4}(\theta)
$$

Then it follows from the compatibility relation (6.1) that

$$
\theta \theta_{x^{5}}=O_{A, 4}(\theta) \text {. }
$$

Dividing $\theta$, we get a regular equation for $\theta$.

Now we consider the KdV equation. The first four steps gave us the following 24 expansions

$$
\begin{aligned}
\theta_{x}^{2}= & 1+O_{A, 9}\left(\theta^{2}\right), \\
\theta_{x^{2}}= & -\frac{\theta \theta_{t} \theta_{x}}{4}+O_{A, 9}\left(\theta^{2}\right), \\
\vdots & \\
\theta_{x t^{6}}= & \frac{715 \theta_{t}^{9}}{32}-\frac{105 \theta_{t^{2}}^{2} \theta_{t^{3}}}{4}-\frac{35 \theta_{t} \theta_{t^{3}}^{2}}{2}-\frac{105 \theta_{t} \theta_{t^{2}} \theta_{t^{4}}}{4}-\frac{21 \theta_{t}^{2} \theta_{t^{5}}}{4} \\
& +\frac{675 \theta_{t}^{4} \theta_{t^{2}}^{2} \theta_{x}}{4}+85 \theta_{t}^{5} \theta_{t^{3}} \theta_{x}+45 \theta_{t}^{2} \theta_{t^{2}}^{2} \theta_{x^{5}}+20 \theta_{t}^{3} \theta_{t^{3}} \theta_{x^{5}} \\
& +\frac{41 \theta_{t}^{7} \theta_{x} \theta_{x^{5}}+60 \theta_{t}^{3} \theta_{t^{2}} \theta_{x^{5} t}+15 \theta_{t}^{4} \theta_{x^{5} t^{2}}-14 \theta_{t}^{6} \theta_{x^{7}}}{4}-\frac{105 \theta_{t^{2}} \theta_{t^{5}}}{32}-\frac{153 \theta_{t} \theta_{t^{6}}}{160} \\
& +\theta\left(-\frac{188397 \theta_{t}^{7} \theta_{t^{2}}}{256}-\frac{175 \theta_{t^{3}} \theta_{t^{4}}}{32}-\frac{1211 \theta_{t}^{2} \theta_{t^{2}}^{3} \theta_{x}}{64}+\frac{17911 \theta_{t}^{3} \theta_{t^{2}} \theta_{t^{3}} \theta_{x}}{32}+\frac{1577 \theta_{t}^{4} \theta_{t^{4}} \theta_{x}}{64}\right. \\
& \quad+\frac{3 \theta_{x} \theta_{x^{2} t^{6}}}{8}+\frac{11 \theta_{t} \theta_{x^{3} t^{5}}}{20}+\theta_{t}^{2} \theta_{x} \theta_{x^{4} t^{4}}+\frac{75 \theta_{t^{2}}^{3} \theta_{x^{5}}}{8}
\end{aligned}
$$




$$
\begin{aligned}
& +32 \theta_{t} \theta_{t^{2}} \theta_{t^{3}} \theta_{x^{5}}+\frac{45 \theta_{t}^{2} \theta_{t^{4}} \theta_{x^{5}}}{8}+\frac{2735 \theta_{t}^{5} \theta_{t^{2}} \theta_{x} \theta_{x^{5}}}{16} \\
& +48 \theta_{t} \theta_{t^{2}}^{2} \theta_{x^{5} t}+\frac{45 \theta_{t}^{2} \theta_{t^{3}} \theta_{x^{5}} t}{2}-\frac{10599 \theta_{t}^{6} \theta_{x} \theta_{x^{5} t}}{16} \\
& +\frac{135 \theta_{t}^{2} \theta_{t^{2}} \theta_{x^{5} t^{2}}}{4}+3 \theta_{t}^{3} \theta_{x^{5} t^{3}}-14 \theta_{t}^{4} \theta_{x} \theta_{x^{6} t^{2}} \\
& \left.-\frac{847 \theta_{t}^{4} \theta_{t^{2}} \theta_{x^{7}}}{8}-14 \theta_{t}^{5} \theta_{x^{7} t}-28 \theta_{t}^{6} \theta_{x} \theta_{x^{8}}\right)+O_{A, 9}\left(\theta^{2}\right)
\end{aligned}
$$

and 4 compatibility relations

$$
\begin{aligned}
0= & \theta^{3}\left(\frac{105 \theta_{t} \theta_{t^{2}} \theta_{x}}{2}+28 \theta_{x^{5} t}+2 \theta_{x^{8}}\right) \\
& +\theta^{4}\left(\frac{455 \theta_{t}^{4}}{16}+70 \theta_{t^{3}} \theta_{x}+42 \theta_{t}^{2} \theta_{x} \theta_{x^{5}}-42 \theta_{x^{5}}^{2}-10 \theta_{t} \theta_{x^{7}}\right)+O_{A, 9}\left(\theta^{5}\right), \\
\vdots & \\
0= & \theta\left(-\frac{315 \theta_{t} \theta_{t^{2}} \theta_{x}}{2}-84 \theta_{x^{5} t}-6 \theta_{x^{8}}\right) \\
& +\theta^{2} \quad\left(-\frac{525 \theta_{t}^{4}}{8}-140 \theta_{t^{3}} \theta_{x}-168 \theta_{t}^{2} \theta_{x} \theta_{x^{5}}+168 \theta_{x^{5}}^{2}\right. \\
& \left.\quad+12 \theta_{t} \theta_{x^{7}}-2 \theta_{x} \theta_{x^{9}}\right)+O_{A, 9}\left(\theta^{3}\right) .
\end{aligned}
$$

The first and the last compatibility relations correspond to the resonance derivatives $\theta_{x^{5}}$ and $\theta_{x^{7}}$. The middle two relations correspond to the "secondary" resonance derivatives $\theta_{x^{5} t}$ and $\theta_{x^{5} t^{2}}$ and are consequences of the first and the last relations.

Now we are ready to construct a regular tenth order equation for $\theta$ for the $\mathrm{KdV}$ equation. We use the indicial normalization $u=\theta^{-2}$ to change the $\mathrm{KdV}$ equation to a third order singular equation for $\theta$. Then we apply $D_{x^{7}}$ and get a tenth order singular equation for $\theta$. We substitute the 24 expansions into this tenth order equation. After simplifying, we get

$$
\begin{aligned}
\theta^{2} \theta_{x^{10}}= & -\frac{315 \theta_{t} \theta_{t^{2}} \theta_{x}}{4}-42 \theta_{x^{5} t}-3 \theta_{x^{8}} \\
& +\theta\left(\frac{105 \theta_{t}^{4}}{16}+70 \theta_{t^{3}} \theta_{x}-168 \theta_{t}^{2} \theta_{x} \theta_{x^{5}}+168 \theta_{x^{5}}^{2}\right. \\
& \left.-30 \theta_{t} \theta_{x^{7}}-5 \theta_{x} \theta_{x^{9}}\right)+O_{A, 9}\left(\theta^{2}\right)
\end{aligned}
$$

Dividing $\theta^{3}$ and $\theta$ from the first and the last compatibility relations, we get

$$
\begin{aligned}
0= & \frac{105 \theta_{t} \theta_{t^{2}} \theta_{x}}{2}+28 \theta_{x^{5} t}+2 \theta_{x^{8}} \\
& +\theta\left(\frac{455 \theta_{t}^{4}}{16}+70 \theta_{t^{3}} \theta_{x}+42 \theta_{t}^{2} \theta_{x} \theta_{x^{5}}-42 \theta_{x^{5}}^{2}-10 \theta_{t} \theta_{x^{7}}\right)+O_{A, 9}\left(\theta^{2}\right) \\
0= & -\frac{315 \theta_{t} \theta_{t^{2}} \theta_{x}}{2}-84 \theta_{x^{5} t}-6 \theta_{x^{8}} \\
& +\theta\left(-\frac{525 \theta_{t}^{4}}{8}-140 \theta_{t^{3}} \theta_{x}-168 \theta_{t}^{2} \theta_{x} \theta_{x^{5}}+168 \theta_{x^{5}}^{2}\right.
\end{aligned}
$$




$$
\left.+12 \theta_{t} \theta_{x^{7}}-2 \theta_{x} \theta_{x^{9}}\right)+O_{A, 9}\left(\theta^{2}\right)
$$

Adding 6 times (6.4) and 5/2 times (6.5) together, we have

$$
\begin{aligned}
0= & -\frac{315 \theta_{t} \theta_{t^{2}} \theta_{x}}{4}-42 \theta_{x^{5} t}-3 \theta_{x^{8}} \\
& +\theta\left(\frac{105 \theta_{t}^{4}}{16}+70 \theta_{t^{3}} \theta_{x}-168 \theta_{t}^{2} \theta_{x} \theta_{x^{5}}+168 \theta_{x^{5}}^{2}-30 \theta_{t} \theta_{x^{7}}-5 \theta_{x} \theta_{x^{9}}\right)+O_{A, 9}\left(\theta^{2}\right) .
\end{aligned}
$$

Combining this with (6.3), we see that $\theta$ satisfies a tenth order regular equation.

In general, if $n$ is the order of the equation and $\theta_{x^{j+1}}$ is the highest order resonance derivatives, then we would expect a regular $(n+j+1)$ order equation for $\theta$.

The obvious drawback of the alternative algorithm is its complexity. As a matter of fact, we can hardly write down the whole equation explicitly. Despite the practical problem, the algorithm does have some theoretical advantage.

Note that the high order regular equation we get for the Burger's equation and the $\mathrm{KdV}$ equation are in the form that we can apply Cauchy-Kowalevski theorem. As a matter of fact, we have also done the computation for the following important equations: $\mathrm{mKdV}, \mathrm{cKdV}$, Boussinesq, KP, Sine-Gordon, Mikhailov, Liouville. In all the cases, we can apply the Cauchy-Kowalevski theorem to the high order regular equation for $\theta$. Consequently, by an argument similar to the one in Section 5 , we have a conceptual proof that the formal Laurent series solutions for these equations are always convergent.

We have shown in [10] that successfully carrying out the algorithm in Section 2 is equivalent to passing the Painlevé test. We believe this also holds for the alternative algorithm. If this can be rigorously proved, then we have a conceptual proof that the formal Laurent series solutions arising from successful application of the Painlevé test to PDEs are always convergent.

\section{REFERENCES}

[1] M. J. Ablowitz And P. A. Clarkson, Solitons, Nonlinear Evolutions and Inverse Scattering, Cambridge University Press, Cambridge, 1991.

[2] M. J. Ablowitz, A. Ramani and H. Segur, A connection between nonlinear evolution equations and ordinary differential equations of P-type I, II, J. Math. Phys. 21: 715-721, 10061015(1980).

[3] A. A. Briot and J.C. Bouquet, Recherches sur les proprietés des fonctions definies par des equations differentielles, J. Ecole Imp. Polytech. 21: 113-197(1856).

[4] L. FuChs, Über differentialgleichungen deren intégrale feste verzweigungspunkte besitzen, Sitz. Akad. Wiss., Berlin 32: 669-720(1884).

[5] R. Fuchs, Sur quelques équations différentielles linéaires du second ordre, Comptes-Rendus Acad. Sc. Paris 141: 555-558(1905).

[6] B. Gambier, Sur quelques équations différentielles du second ordre et du premier degre dont l'intégrale générale est à points critiques fixés, Acta. Math. 33: 1-55(1909).

[7] B. Grammaticos and A. Ramani, Painlevé integrability: theorems and conjectures, In: Painlevé Transcendents: Their Asymptotics and Physical Applications, ed. by D. Levi and P. Winternitz, Plenum Press, New York and London, 145-162(1992).

[8] P. HoYeR, Über die Integration eines Differentialgleichungsystems von der Form

$$
\begin{aligned}
\frac{d x_{1}}{d t} & =a_{1} x_{2} x_{3}+a_{2} x_{3} x_{1}+a_{3} x_{1} x_{2} \\
\frac{d x_{2}}{d t} & =b_{1} x_{2} x_{3}+b_{2} x_{3} x_{1}+b_{3} x_{1} x_{2} \\
\frac{d x_{3}}{d t} & =c_{1} x_{2} x_{3}+c_{2} x_{3} x_{1}+c_{3} x_{1} x_{2}
\end{aligned}
$$

durch elliptische Funktionen, Dissertation Kongl. Friedrich-Wilhems, Univ. in Berlin 1$36(1879)$. 
[9] J. Hu And M. Yan, Singularity analysis for integrable systems by their mirrors, 1998.

[10] J. Hu AND M. YAN, Analytical aspects of the Painlevé analysis, 1998.

[11] N. Joshi And M. Kruskal, A direct proof that solutions of the six Painlevé equations have no movable singularities except poles, Stud. Appl. Math. 93: 187-207(1994).

[12] N. Joshi and J. A. Petersen, A method of proving the convergence of the Painlev expansions of partial differential equations, Nonlinearity 7: 595-602(1994).

[13] N. Joshi And J. A. Petersen, Complex blow-up in Burgers' equation: an iterative approach, Bull. Austral. Math. Soc. 54: 353-362(1996).

[14] N. Joshi and G. K. SRinivasan, The radius of convergence and the well-posedness of the Painlev expansions of the Korteweg-de Vries equation, Nonlinearity 10: 71-79 (1997).

[15] S. Kowalevski: Sur le probléme de la rotation d'un corps solide autour d'un point fixé, Acta Math. 12: 117-232(1889).

[16] J. B. McLeod And P. J. Olver, The connection between partial differential equations solvable by inverse scattering and ordinary differential equations of Painlevé type, SIAM J. Math. Anal. 14: 488-506(1983).

[17] P. Painlevé, Sur les équations différentielles du premier ordre, Comptes-Rendus Acad. Sc. Paris 107: 221-224, 320-323, 724-726(1888).

[18] P. PAinlevé, Mémoires sur les équations différentielles dont l'intégrale générale est uniforme, Bull. Soc. Math. France 28: 201-261(1900).

[19] P. PAinlevé, Sur les équations différentielles du second ordre à points critiques fixès, Acta Math. 25: 1-85(1902).

[20] J. Weiss, M. Tabor and G. Carnevale, The Painlevé property for partial differential equations, J. Math. Phys. 24: 522-526(1983). 